\title{
Upcoming challenges in intellectual property presented by emerging pharmaceutical technologies
}

\author{
"In order to analyze humans as a collection of (biological) species \\ and to elucidate the respective individual properties such that \\ personalized and tailored treatments can be provided, it is necessary \\ to use big data analysis."
}

First draft submitted: 10 November 2016; Accepted for publication: 9 December 2016; Published online: 15 February 2017

Keywords: Al • artificial intelligence $\bullet$ big data $\bullet$ intellectual property $\bullet$ IP $\bullet$ patent

$\bullet$ personalized medicine $\bullet$ precision medicine $\bullet$ regenerative medicine $\bullet$ stem cell

\section{Emerging pharmaceutical technologies: some intellectual property concerns}

The NIH defines precision medicine (also known as personalized medicine) as "an emerging approach for disease treatment and prevention that takes into account individual variability in genes, environment, and lifestyle for each person" [1].

During Barack Obama's State of the Union Address [2] on 20 January 2015, it highlighted that the government's commitment to investing in precision medicine:

\section{"[So] tonight, I'm launching a new}

Precision Medicine Initiative to bring us closer to curing diseases like cancer and diabetes, and to give all of us access to the personalized information we need to keep ourselves and our families healthier. We can do this."

The USA is the global leader in the pharmaceutical industry, and the government's plan to boost investment in precision medicine reflects current trends worldwide. This editorial was prepared just after the US Presidential Election 2016 and so at this stage it is uncertain whether this policy will be impacted, although, in my opinion, it is unlikely that the new President will make any significant amendments.
There are a number of issues related to precision medicine in terms of intellectual property (IP) rights, particularly as precision medicine draws on a number of emerging pharmaceutical technologies. In this editorial, I have selected to discuss current and potential issues related to intellectual property rights in the fields of big data and artificial intelligence (AI), and regenerative medicine, which constitute areas that are receiving increased attention in view of the growing emphasis on precision medicine.

\section{Harnessing big data \& Al}

In order to analyze humans as a collection of (biological) species and to elucidate the respective individual properties such that personalized and tailored treatments can be provided, it is necessary to use big data analysis. Big data refers to a collection of data that cannot be processed in a realtime manner using conventional database applications, and are typically described as having the three Vs: volume, variety and velocity, and sometimes two additional Vs: veracity and value.

In 2017, it is predicted that intellectual property in relation to big data will become a hot topic in pharmaceutical industry. This

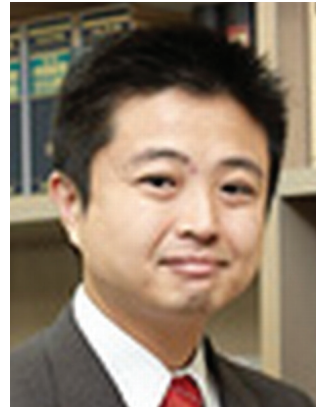

Takeshi S Komatani Principal Patent Attorney, SHUSAKU.YAMAMOTO, 17th Floor Grand Front Osaka Tower, C 3-1 Ofuka-cho, Kita-ku, Osaka 5300111, Japan

tskomatani@shupat.gr.jp 
is reflected in the agendas of upcoming symposia and conferences, such as the international conference on 'Big Data Analytics in Pharma', which will be held in Brussels (Belgium) in February 2017 [3]. Most big pharma companies have introduced an informatics division dealing with big data, in order to facilitate $\mathrm{R} \& \mathrm{D}$, as it is widely acknowledged that big data analytics may provide a way to harness unleveraged and/or underleveraged data and gain timely insights for making better business decisions.

"In line with the rise in the use of artificial intelligence in pharmaceutical sciences, and other industries, the Japanese government have this year announced that they are considering establishing a new protection system for intellectual property...

In dealing with big data, $\mathrm{AI}$ is also garnering attention among pharmaceutical industry. In March 2016, a Baltimore start-up company, Insilico Medicine, announced that they had formed the Pharmaceutical Artificial Intelligence division [4]. As mentioned in their press release, AI may be used in relation to "transcriptomics-, proteomics-, blood biochemistry-based biomarkers of multiple diseases, predictors of alternative therapeutic uses of multiple drugs and analytical tools for high-throughput screening," such that AI can be applied to drug discovery and development by introducing deep learning. Big data's applications are not limited to the deep learning, and can be applied to personalized medicine, by gathering 'global' information from the respective individuals. AI 'doctors' such as IBM Watson, a self-learning AI tool, are now able to analyze big data including different types of data such as medical literature, patents, genomics and chemical and pharmacological data to look for a new and/or alternative therapies (e.g., see the case of IBM Watson [5]). With this in mind, we may predict that one day big data and AI may be widely used by clinicians to assist in medical consultations in future hospitals and clinics.

In line with the rise in the use of $\mathrm{AI}$ in pharmaceutical sciences, and other industries, the Japanese government have this year announced that they are considering establishing a new protection system for intellectual property created by a nonhuman, for example, AI, which was proposed in the 'Intellectual Property Promotion Plan 2016' and approved at a meeting held at the Japanese Intellectual Property Strategy Headquarters on 9 May 2016 [6,7]. The plan states that, "moving forward, the government will continue to consider the extent of intellectual property rights and for whom they will be granted, in relation to creative works produced by artificial intelligence." Other governments in the world are also considering how to deal with such intellectual property.

The rise of AI may also result in other serious issues from legal point of view. Currently, natural persons assume legal, in other words, criminal and/or civil responsibility for results of their act, which may lead to criminal charges or liability for damages. In addition, legal persons such as pharmaceutical companies are now deemed to assume similar legal responsibility. However, unlike pharmaceutical companies, AI cannot be regarded as a responsible subject based on the current legal system. This issue is most prominent in automobile industry, since automatic driving vehicles are now available in the real world. In 2016, there was a symposium entitled "Legal dimensions of Big Data in the Health and Life Sciences - From Intellectual Property Rights and Global Pandemics to Privacy and Ethics" at the University of Copenhagen $[8]$, in which, not only intellectual property, but also privacy and ethics were discussed, as nowadays, one cannot ignore the intersection of intellectual property and global public health. Advances in precision medicine, drug repurposing and applications of AI in the sense of machine learning in big medical datasets which have followed from the big data revolution, were also discussed, and, in relation to this, limitations in intellectual property, such as patents and trade secrets (see, e.g., a report from an IP law firm in the USA [9]). In the future, practitioners have to find a way to efficiently and effectively protect new types of innovation based around big data and AI using the existing system, something which I believe is within reach.

It is also important to consider that big data and AI generally concern open and transparent innovations. Namely, the regulation, governance and use of biological, genetic and personal data need to be reconsidered from a legal point of view, specifically focusing on public-private research collaborations, data integrity, privacy and ethics in terms of intellectual property law, competition law, R\&D incentives and commercialization. In a recent article by Michael Carroll, limitations of patent law, copyright law and laws of trade secrecy are discussed in terms of issues of sharing research [10].

\section{Regenerative medicine \& stem cell technologies: ultimate weapon or a working progress?}

In recent decades, regenerative medicine and stem cell technologies have become increasingly established and have a potential to enhance precision medicine. For example, a patient's own cells (not restricted to stem cells) can be isolated and optionally 'reprogrammed' 
to stem cells, and properly differentiated to the tissue of interest. As such, some approaches to precision medicine involve such stem cells production, engineering tissue therefrom and returning the tissue to the patient (see, e.g., University of Utah's website [11]). In Japan, the Pharmaceutical Affairs act was amended in 2014 [12] to introduce regenerative medicine products. In the new act, regulations on drugs, medical devices and regenerative medicine products were divided into individual chapters to restructure the entire framework. The name itself was also amended from the Pharmaceutical Affairs Law to the Law for ensuring quality, efficacy and safety of drugs and medical devices. With respect to regenerative medicine, a 'provisional' approval or 'approval with conditions and time limit' system has been introduced. According to the new system, nonhomogenous quality tissueengineered medical products can be approved earlier than with the conventional (regular) approval system with conditions and time limit if they are assumed to be effective and proven to be safe in humans. The applicant is required to verify the efficacy and safety and resubmit the application within ' 7 years' after the conditioned approval. As such, drug development based on regenerative medicine is accelerated in Japan.

Although mainly used in therapies and treatments, stem cell technologies are also used as a tool for drug discovery and development using stem cells such as induced pluripotent stem cells, pharmaceutical companies can simulate a number of biological conditions, which can be used to perform tests which are required for market authorization/approval, especially in preclinical research.

From an intellectual property point of view, the regenerative medicine community is faced with a difficult problem. Namely, the best quality of regenerative medicine is $100 \%$ identical property to the natural, healthy conditions (or [healthy] human). In this regard, in the USA, the Supreme Court held a decision in which limitations of patent-eligible subject matter with respect to the nature-based products should be strictly considered. Thereafter, the US Patent and Trademark Office has revised its guidelines (Manual of Patent Examining Procedure) and proposed two-step tests for judging patent-eligible subject matter [13]. In brief, step 1 determines whether the claim is directed to a process, machine, manufacture or composition of matter. This is a requirement based on the statute and is not changed from before. Step 2 is the two-part analysis from Alice Corp., also known as the Mayo test, for claims directed to laws of nature, natural phenomena and abstract ideas (the judicially recognized exceptions). In step $2 \mathrm{~A}$, "determine whether the claim is directed to a law of nature, a natural phenomenon, or an abstract idea (judicial exceptions). If no, the claim is eligible and examination should continue for patentability. If yes, proceed to step $2 \mathrm{~B}$ to analyze whether the claim as a whole amounts to significantly more than the exception." If the regenerative medicine with 'the best quality', in other words, a product which is entirely identical to a natural product, is examined, it is highly likely that such products will fall within 'claims directed to laws of nature and natural phenomena'. Therefore intellectual property practitioners must consider this limitation and consider the best course of action in protecting regenerative medicine innovations. This limitation may affect how to advance regenerative medicine R\&D in pharmaceutical industry. Namely, if sufficient intellectual property protection is not secured for the regenerative medicine and stem cell technologies, there will be a lower number of investors who wish to invest in regenerative medicine $R \& D$ in pharmaceutical industry.

\section{7 \& beyond}

I have touched upon several key topics for 2017 in this editorial. Although the technologies discussed are not overly novel, in the coming year, it is likely that they will finally be put into practice and made a reality, such as the new version of Watson [5], IBM's AI. Therefore, it is expected that such cognitive computing technology will represent a real game changer in pharmaceutical R\&D in 2017. In terms of precision medicine, AI/big data and regenerative medicine/stem cell technologies are just examples of many emerging technologies in this area. Namely, genetic technologies for finding the proper dose of the drug, genomic technologies for improving treatment of genetic diseases such as cancers, molecular profiling of microbes including disease-causing bacteria, fungi and viruses, technologies relating to daily dietary requirements such as personalized diet plans, among others. However, as touched upon above, these emerging technologies are proving a challenge and have led to a number of intellectual property issues, which are yet to be solved. For example, if - hypothetically AI were deemed a legal person, then AI would also be subject to the possibility of infringement. However, such acts of infringement by an AI, an artificial person, will likely not fit the current legal system. In one of the most extreme cases, there could be some instances, for example, where a US AI judge will proceed with an infringement lawsuit between a German AI proprietor and a Japanese AI potential infringer, based on UK law. However, nobody has given a clear answer if this will happen and/or how this will be proceeded. 


\section{Conclusion}

In this editorial, I have discussed a number of aspects that are or will be active in the pharmaceutical industry. Most of the technologies touched upon were actually born several or tens of years ago; however, they have not yet become a reality. I believe that in the year 2017 we will witness many of these technologies finally being put into practice, likely bringing with it IP issues, that in turn call for game-changing patent portfolios and advanced strategy.

\section{References}

1 All of USSM research program, a NIH's website. About the Precision Medicine Initiative Cohort Program. www.nih.gov/research-training/allofus-research-program

2 Remarks by the President in State of the Union Address | 20 January 2015. The White House's website. www.whitehouse.gov/the-press-office/2015/01/20

3 Big data analytics in pharma. www.bigdatainpharma.com

4 AAAS EurekAlert! Pharma.AI launches to apply artificial intelligence to drug discovery and development. Press Release. www.eurekalert.org/pub_releases/2016-03/imi-plt031516

5 Chen Y. IBM Watson: how cognitive computing can be applied to big data challenges in life sciences research. Clin. Ther. 38(4), 688-701 (2016).

6 Intellectual Property Strategy Headquarters. Press release. http://japan.kantei.go.jp/97_abe/actions/201605/09article3

\section{Financial \& competing interests disclosure}

The author has no relevant affiliations or financial involvement with any organization or entity with a financial interest in or financial conflict with the subject matter or materials discussed in the manuscript. This includes employment, consultancies, honoraria, stock ownership or options, expert testimony, grants or patents received or pending, or royalties.

No writing assistance was utilized in the production of this manuscript.

7 Komatani TS. E-News No. 47, IP of AI - New type of intellectual property? How the TRIPS Agreement is involved? http://aippi.org/ip-of-ai

8 University of Copenhagen, Global Genes Local Concerns. Legal dimensions of Big Data in the Health and Life Science. http://globalgenes.ku.dk/calender

9 Jason L. Artificial intelligence drives new thinking on patent rights, LimeGreen IP News, Hogan Lovells US LLP, 2016. www.limegreenipnews.com/2016/07/artificial-intelligence

10 Carroll MW. Sharing research data and intellectual property law: a primer. PLoS Biol. 13(8), e1002235 (2015).

11 More Examples of Precision Medicine in Action. http://learn.genetics.utah.edu/content/precision/action

12 Japanese Law No. 103 dated 13 December 2013.

13 United States Patent and Trademark Office, USPTO. 2014 Interim Guidance on Subject Matter Eligibility. www.uspto.gov/patent/laws-and-regulations 